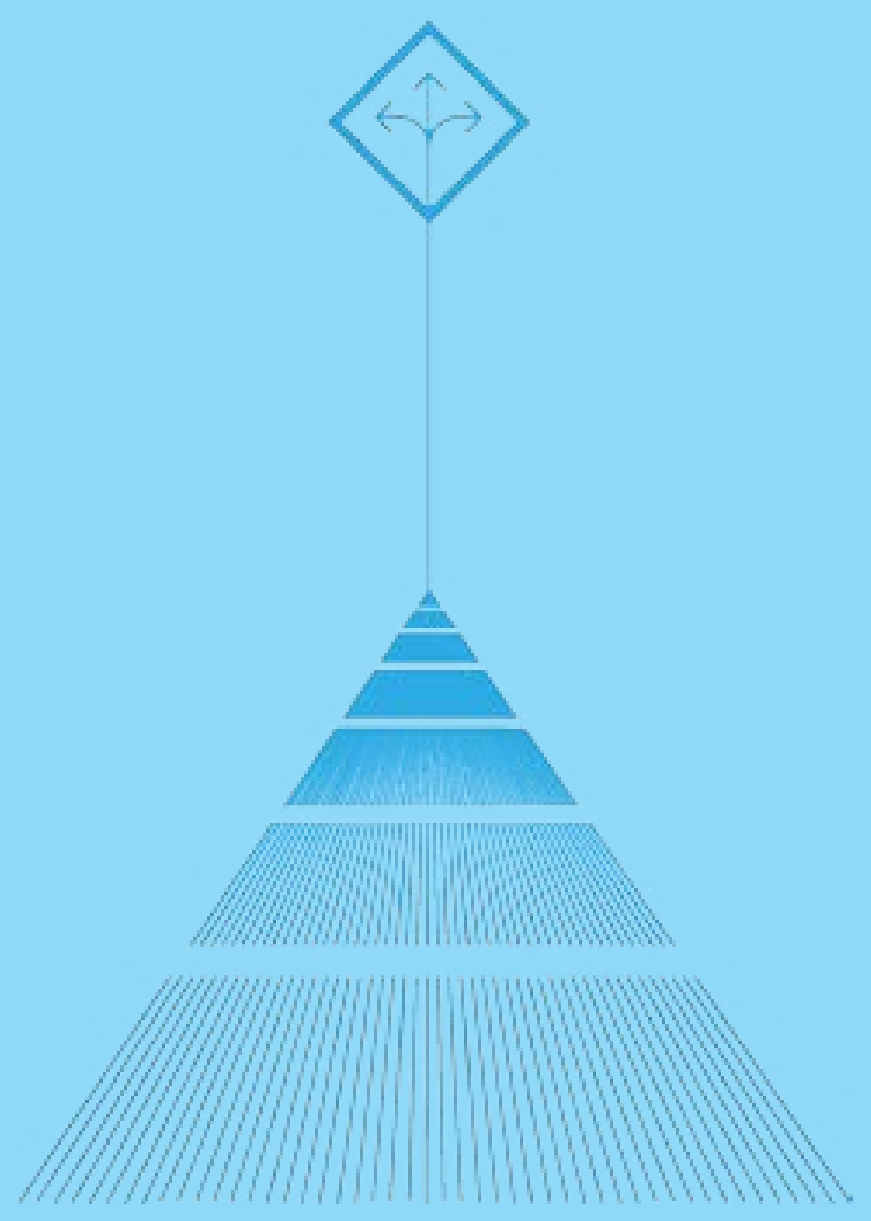

\title{
La identidad profesional del profesor en educación secundaria. El posicionamiento político en la función del maestro
}

Professional identity of the teacher in the secondary school. Political position on the teacher's role

Identidade profissional na secundário formação de professores.

Posição político sobre o papel do professor

Francisco Hernando Gómez Torres / Elizabeth Magaly Niño Gutiérrez 


\section{Francisco Hernando Gómez Torres ${ }^{1}$ \\ Elizabeth Magaly Niño Gutiérrez²}

1 Candidato a Doctor en Psicología de la Educación, Universidad Autónoma de Barcelona. Magíster en Psicología de la Educación de la Universidad de Barcelona; Máster en Educación de la Universidad Santo Tomás. Investigador del grupo identitES de la Universidad Autónoma de Barcelona y del grupo Filosofía, pensamiento y sociedad, de la Corporación Universitaria Minuto de Dios. Profesor de planta en el Departamento de Filosofía de la Corporación Universitaria Minuto de Dios. Correo electrónico: fgomeztorres@yahoo.com

2 Magíster en Educación de la Universidad Santo Tomás; Profesora del CED Antonio José de Sucre y de la Licenciatura en Pedagogía Infantil de la Corporación Universitaria Minuto de Dios. Correo electrónico: emnino46@yahoo.com.mx

Fecha de recepción: 12 de Febrero de 2015 / Fecha de aprobación: 3 de abril de 2015.

\section{Resumen}

Tomando como lente la identidad profesional, en esta investigación se explora el posicionamiento político del profesor en la educación secundaria, a partir del rol como unidad de análisis. A través de un examen de carácter cualitativo, de enfoque fenomenográfico, se indagó, mediante entrevista semiestructurada, a 20 profesores de dos campos disciplinares, Ciencias Sociales y Matemáticas, sobre las atribuciones asociadas a la función profesional, encontrando que existen dos formas en común para entender la actividad: como formadores y orientadores. Los resultados indican que hay determinados posicionamientos en el rol docente, cuyo énfasis se asocia a la perspectiva de política para la vida o del estilo de vida, como una dimensión individual de la política (Giddens, 1991).

\section{Palabras clave}

Identidad profesional, rol docente, política para la vida.

\section{Summary}

Taking professional identity like eyeglass, in this research is explored the political positioning of teachers in secondary school beginning with the teacher's role as unit of analysis. Through a qualitative research, of phenomenographic approach was investigated by semistructured interview to 20 teachers in two disciplinary areas: Social Sciences and Mathematics, on the functions associated with the professional role, finding that there are two common ways understanding activity: as former and tutor. The results indicate that there are certain positions on the teacher's role whose emphasis is associated with the political perspective or lifestyle, as an individual dimension of politics (Giddens 1991).

\section{Key words:}

Professional identity, teacher's role, lifestyle political.

\section{Resumo}

Tomando identidade profissional como óculos, nesta pesquisa é explorado o posicionamento político dos professores na escola secundária que começa com o papel do professor como unidade de análise. Por meio de uma pesquisa qualitativa, de abordagem fenomenográfica foi investigado por meio de entrevista semi-estruturada para 20 professores em duas áreas disciplinares: Ciências Sociais e Matemática, sobre as funções associadas à função profissional, descobrindo que há duas maneiras comuns atividade compreensão: o ex e tutor. Os resultados indicam que há certas posições sobre o papel do professor, cuja ênfase está associada à perspectiva política ou estilo de vida, como uma dimensão individual da política (Giddens, 1991).

Palavras chave:

Identidade profissional, o papel do professor, de estilo de vida política. 


\section{Introducción}

$\mathrm{L}^{2}$ a identidad profesional del profesor es un área de estudio que se incorpora al campo de la investigación educativa desde los años noventa (Beijar, Meijer y Verloop, 2004); ayuda a entender la complejidad de la práctica docente y propone alternativas para realizar innovaciones educativas encaminadas a la formación del profesorado (Enyedy, Godberg y Muir 2005; Beuchamp y Thomas, 2009). Se considera como una lente (Gee, 2000) que permite indagar, sobre la satisfacción laboral, la motivación y el compromiso hacia la profesión (Settlage, Southerland, Smith y Ceglie, 2009) y la valoración sobre la eficacia en la actuación comparando las potencialidades percibidas por el profesor, con las metas a lograr en el ejercicio de la actividad profesional (Day, Kington, Stobart y Sammons, 2006). En este caso, su relación con el posicionamiento político.

Esta relación se ha explorado desde el impacto de las políticas educativas y la gestión institucional, en el sentido que dan los profesores a su labor, identificando las disyuntivas entre las exigencias establecidas mediante los mandatos legales frente a las creencias y expectativas de los profesores al agenciar su trabajo (Galaz, 2011; Salazar, Valenzuela, Cortes y Guillen, 2013; Arriaga, 2013; Lasky, 2005; Stillwagon, 2008).

De los estudios consultados, asumimos que las políticas educativas aparecen como herramientas culturales que tienen impacto en la identidad docente (Wertsch, 1991); lo que los individuos creen y piensan sobre cómo deben actuar, se forma por las estructuras culturales, históricas y sociales de las que hace parte la política como mediador con incidencia en el funcionamiento cognitivo y en la actuación del maestro.

La actividad del profesor, como conjunto de acciones mediadas por la política, implica que a pesar del gobierno normativo e institucional al que somete al maestro, él puede ser capaz de un posicionamiento político, en el sentido de manifestar creencias y disposiciones que se vinculan al fin último de su función social, su profesión y su trabajo; esta posición se evidencia en los roles que se atribuyen en un contexto y exigen a este profesional trabajar no solo en la enseñanza y el aprendizaje de un saber, sino en diversos asuntos como: formar en hábitos, formar ciudadanos, formar en campos especializados del conocimiento, asesorar a la familia, atender a población con discapacidad, orientar sexualmente y en situaciones conflictivas que afectan la convivencia.
La política se puede considerar como un factor que incide en la constitución de la identidad profesional del profesor (Galaz, 2011) y en la gestión docente; participa como parte de las influencias de tipo ideológico y cultural que la construyen (Salazar, et al., 2013; Galaz, 2011; Weise, 2011; Stillwagon, 2008; Zembylas, 2005 y Lasky, 2005). Así, inicialmente presentamos la perspectiva identitaria asumida y luego el papel de los roles, como dimensión de la identidad y unidad de análisis, para aproximarse al posicionamiento político del profesor; finalmente, se da una mirada a la manera en que se aborda la figura del maestro en su dimensión política.

\section{La identidad profesional del profesor}

La identidad se ha estudiado desde diferentes perspectivas, Akkerman y Meijer (2010) han distinguido dos visiones. La primera de carácter determinista e individual, sustentada en el objetivismo; la segunda mantiene una visión relativista y social. Entre ambas, las posiciones son diversas: De la identidad unitaria a una visión múltiple, de la idea de permanencia y continuidad a una visión de cambio constante, y de una construcción esencial a una identidad socialmente construida (Weise, 2011).

Asumimos que la identidad se construye de manera dialógica y negociada (Hermans, 2001), pasa por procesos de construcción y de reconstrucción de significados a partir de la propia experiencia, y se revela a través de un conjunto de representaciones que pueden ser más o menos estables (Monereo, 2010), pero dependientes de ámbitos de socialización (Galaz, 2011; Akkerman y Meijer, 2010; Day, 2006; Monereo y Badia, 2011).

La identidad contiene creencias sobre los roles a desempeñar profesionalmente, su origen es contextual, por lo que las creencias de los profesores están mediadas por las estructuras culturales e históricas y por las políticas educativas que influyen en el sentido atribuido a la profesión (Lasky, 2005). A través de la organización escolar el sistema educativo gestiona y legitima intereses establecidos mediante la legislación, la escuela se define por un compuesto de reglas que guían la acción (Zucker, 1987) y el docente desarrolla su vida profesional en ese contexto.

Frente a lo expuesto, no hay certeza sobre la manera en que la identidad profesional del profesor en educación secundaria es afectada por las mediaciones políticas en el contexto educativo colombiano, pero sabemos que toda reforma educativa se 
desarrolla bajo los mismos esquemas existentes, la misma infraestructura, organización y jerarquía escolar (Arriaga, 2013), sin apreciar el impacto que puede tener en la manera que el maestro percibe la profesión y cómo debe llevarla.

Creemos que al aproximarse a la identidad narrada a través del rol es posible identificar un posicionamiento político, una distinción entre el ser gobernado y la capacidad de acción política. De acuerdo con Lasky (2005), la agencia está siempre mediada por la interacción entre el individuo, sus atributos e inclinaciones y las herramientas y estructuras del entorno, lo que puede hacer un profesor es adaptar, adoptar o ignorar el mandato.

\section{El rol como componente de la identidad}

Existe un contraste en la forma de entender el rol del maestro, por una parte, como una asignación de desempeños y competencias esperadas en el ejercicio profesional, sujeto a las imposiciones políticas sobre lo que debe hacer; por la otra, como imagen de sí mismo en tanto profesional de la educación, constituida por la idea acerca de la función a ejercer (Settlage, Southerland, Smith y Ceglie, 2009; Cohen, 2010). Así, el rol, más que tarea asignada, corresponde a atribución de sentido (Badía y Álvarez, 2010) en relación con lo que se hace durante el ejercicio de la profesión y la responsabilidad a cumplir.

En esta investigación se considera que las atribuciones sobre el rol implican un posicionamiento del profesor que está relacionado con el propósito de la actividad profesional; por ende, ubicados en la segunda perspectiva, siguiendo a Weise (2011), es posible pensar que hay un proceso de negociación entre esa asignación y la "realidad educativa", que depende de decisiones sobre a quién y cómo beneficia el ejercicio profesional, a lo que se pueden vincular otros factores.

Las investigaciones muestran que la percepción acerca de los estudiantes y sus condiciones sociales, así como los factores instruccionales o del aula (Badía y Gómez, 2014) son la fuente de la mayoría de creencias acerca de los roles. Unos se vinculan a la interacción con los estudiantes (Ben-Peretz, Mendelson, Nili y Kron, 2003), a sus habilidades y características diferenciales (Waeyten, Lens y Vandenberghe, 2002) e incluso a la planeación y desarrollo de la actividad educativa. Antes y después de dar una clase el profesor puede entender su tarea como expositor, tutor $\mathrm{u}$ orientador (Nargund-Joshi, Rogers y Akerson, 2011).
Otros factores, como el tipo de disciplina y el nivel escolar, influyen en la tarea asumida. En cuanto a la disciplina, se han encontrado diferencias en la atribución sobre el rol en profesores que enseñan matemáticas (Gil y Rico, 2003) y en los que enseñan ciencias (Carvajal y Gómez, 2002) y en relación con el nivel escolar en educación secundaria. Putnam y Borko (2000), citados por Monereo y Monte (2011), encuentran que los docentes suelen considerar que su función consiste en transmitir un saber disciplinar (rol de experto en una materia); en dotar al alumno de ideas, procedimientos y recursos para indagar (rol de guía); o en educar al alumno en un conjunto de hábitos de conducta y valores (rol de educador).

Entre los niveles educativos hay diferencias con relación al papel a representar, en el contexto de la secundaria hay un conjunto distintivo de circunstancias frente a los alumnos, las políticas educativas y condiciones sociales de los participantes, que inciden en la ecología del trabajo, un poco diferente a la que se encuentra en los niveles elementales (Trickett, Rukhotskiy, Jeong, Genkova, Oberoi, Weinstein y Delgado, 2012).

Lo expuesto da a entender, frente a la gobernabilidad institucional e incluso del sistema educativo que delimita las actuaciones posibles del profesor (Van Veen, Sleegers y Van de Ven, 2005), que los roles, como atribuciones sobre la función, emergen como una política del estilo de vida (Giddens, 1991), es decir, como intereses de actuación vinculados a un contexto, orientaciones sobre el fin de la actividad docente, que van más allá de la función formal establecida por las diferentes instancias (Trickett, et. al., 2012).

Así, el rol es una manifestación de la individualidad que implica la adopción de estilos de vida elegidos libremente, permitiendo un mayor sentido de autenticidad en la trayectoria de vida de las personas en el escenario de una profesión, mostrando que la atribución sobre el sentido de la función docente es a su vez una lectura de lo que considera indispensable, lo que el profesor debe hacer para lograr los objetivos establecidos en el contexto educativo. En educación secundaria, un profesor quizás pueda seguir los estándares y lineamientos definidos, pero también puede seguir los propósitos últimos que nacen de su perspectiva sobre el bien común.

Se habla de múltiples roles porque existen varios mecanismos a través de los cuales un contexto puede dar origen a los mismos; emergen como parte del sentido que el profesor asigna a las relaciones con otros (Levin y He, 2008), a las condiciones de la situación educativa que dan forma a la naturaleza de las acciones 
del profesor, de allí que O’Connor (2008) lo entienda como un conjunto de experiencias de naturaleza social y cultural, aceptadas en el ejercicio libre de una persona, y que Novoa (2000) se refiera al rol en términos de definiciones sociales, políticas, normas, reglas, creencias, convicciones y verdades acerca de lo que significa ser un buen maestro.

Definimos el rol del profesor en la educación secundaria como atribución social que se expresa acerca de lo que hace, emerge de su experiencia en las situaciones educativas y de su formación profesional. Por su carácter contextual y situado, se vincula con exigencias sociales asociadas a la idea de lo que debe hacer en tanto profesional, dándose un proceso de negociación entre asignaciones y atribuciones; en este sentido, es la capacidad de agencia social, de tomar decisiones sobre cómo actuar en relación con el otro, lo que le vincula a la política primera (Giddens, 1991).

\section{La dimensión política en la identidad del profesorado}

No solo el gobierno institucional es suficiente para que una política tenga sentido para el profesor, se ha mostrado cómo, si no se considera al profesor en su capacidad de acción política, el impacto de la legislación y el ambiente de reforma tienen efectos negativos en un sistema educativo. Salazar, Valenzuela, Cortez y Guillen (2013) encuentran que las políticas no alineadas con las creencias y los objetivos de los profesores pueden tener efectos en la satisfacción y las expectativas a futuro. Por su parte, Arriaga (2013) identifica que las políticas educativas para los docentes representan solo ideas que cambian como parte de la política de Estado, ellos las pueden aplicar y aceptarlas o estar en desacuerdo con ellas y rechazarlas. Las disposiciones normativas plantean demandas a los profesores que desafían sus intereses y creencias, pueden aparecer inconsistentes, generando sentimientos de vulnerabilidad y disminuyendo la efectividad en la enseñanza (Weise, 2011).

Se responsabiliza a los profesores de los fracasos en el sistema educativo (Vaillant 2010), sin considerar que las políticas educativas en ocasiones no tienen en cuenta a los docentes, especialmente en el modo de hacer las cosas en el aula; tampoco se ha colocado en el centro del debate, de manera integral, la profesionalización docente, por eso es importante acercarse a la identidad profesional del profesor entendiendo que es un mecanismo mediante el cual se reconoce a sí mismo y, al tiempo, permite a otros reconocerle como miembro de una determinada categoría social, la categoría de profesores (Vaillant, 2010).

Se ha encontrado que mientras las autoridades educativas se esfuerzan en promover competencias didácticas, en realidad los profesores movilizan competencias destinadas a satisfacer los desafíos socio-afectivos que les presentan los estudiantes (Galaz, 2011); esta perspectiva plantea que la educación es un campo de negociación donde diferentes intereses compiten entre sí, el profesor se encuentra entre la gobernabilidad y su capacidad y posibilidad de agencia.

La función docente se puede entender desde la perspectiva de "política de vida o del estilo de vida" (Giddens, 1991) porque en medio de las presiones estructurales del sistema educativo, emerge una forma de individualidad moralmente justificable, una manifestación de decisiones políticas que derivan de la libertad de elección, al tiempo, se desarrollan propuestas como respuesta a la pregunta: ¿Cómo hemos de vivir? Se trata de un sentido de la política que tiene que ver con la experiencia y las prácticas de relación, de mediación y convivencia que sostienen las dimensiones básicas del vivir y convivir, una política que solo se hace en relación con el otro (Bolívar, 2006).

Desde esta perspectiva lo personal es político, el profesor no es títere de la estructura, pero tampoco hace lo que desea, en el proceso realiza ajustes que se manifiestan en su actuación, en la función que dice asumir (Bolívar, 2006), dado que toma decisiones sobre lo que debe hacer, considerando su relación con otros.

Las creencias sobre el rol docente muestran determinados niveles de división del trabajo; si se actúa como maestro, ello implica que no solo se trata de adoptar, resistir o asignar unas actuaciones, los roles son decisiones sobre la función a llevar a cabo, que es resultado visible de las situaciones específicas y de discursos educativos amplios (Kelly, Dorf, Pratt, y Hohmann, 2013). Los procesos y la gobernabilidad del sistema educativo y de la institución definen la tarea docente, a partir de esta tarea, la identidad del profesor comienza a cobrar sentido en el contexto educativo; el sistema sociopolítico influye en la definición de los roles docentes, y ellos hablan acerca de cómo es el sistema educativo.

Al describir el rol se advierten posicionamientos políticos y también el sentido de la actividad profesional, la función da sentido a lo que se hace y se es, el rol aparece como un indicador 
que emerge en el discurso cuando se pregunta al profesor por el fin último de su función. Teniendo en cuenta lo enunciado hasta aquí, nuestra pregunta es: ¿Cómo se posiciona políticamente el profesor en la educación secundaria? Al respecto perseguimos dos objetivos:

- Identificar el sentido que los profesores atribuyen a la función docente, desde la perspectiva identitaria, mediante la clarificación de los roles manifestados.

- Comparar los roles emergentes que aparecen entre profesores de campos disciplinares diferentes (ciencias sociales y matemáticas) al enunciar las funciones atribuidas.

\section{Marco todológico}

La investigación es cualitativa y se desarrolla en el marco de la fenomenografía (Marton, 1994). Se hace una descripción de las funciones atribuidas al ejercicio profesional del profesor en educación secundaria, con el fin de establecer las variaciones surgidas desde la experiencia vivida, enfocándose en lo que percibe y hace para desenvolverse al interior del contexto educativo inmediato (Marton y Pang, 1999).

La unidad de análisis es el rol del profesor, el discurso es la manera de conceptualizar la experiencia profesional. La descripción de la experiencia se reconstruye como "categorías de descripción", la fenomenografía intenta reconocerlas, resultando en una distinción empírica, interpretada, inductiva e idiosincrática de las experiencias que son representadas por los profesores (Akerlind, 2005).

\section{Participantes}

Se contó con la cooperación de 20 profesores, 10 de ciencias sociales y 10 de matemáticas, que imparten la formación entre los grados sexto a once en la ciudad de Bogotá, Colombia. Los criterios para su selección se han establecido por conveniencia, teniendo como criterio la disponibilidad de los colegios y de los participantes.

\section{Recolección y análisis de información}

La investigación en curso, sobre la identidad del profesorado en educación secundaria, utilizó el apartado correspondiente a los roles del profesor que se incluía en una entrevista semi-estructurada, validada a través del juicio de expertos. El análisis se llevó a cabo en "Atlas ti", para lo cual se transcribió, analizó y codificó la información. La técnica empleada fue un análisis de contenido para la identificación de los enunciados; posteriormente, éstos se agruparon en categorías de acuerdo con temáticas comunes, realizando el proceso por codificación abierta y luego axial (Flick, 2007).

\section{Resultados}

En esta investigación los roles expresados por los profesores se revelan como creencias sobre su práctica; proveen una unidad de análisis y se pueden asignar, resistir o adaptar (Kelly, Dorf, Pratt y Hohmann, 2013). Además, se circunscriben a la escuela y ésta se define por un compuesto de reglas que organizan la acción de quienes participan en ella (Salazar, Valenzuela, Cortez y Guillén, 2013). La escuela se supedita a las restricciones del sistema educativo, en este escenario surge una forma de entender la finalidad de la actividad docente. Los roles emergen del intercambio entre las exigencias de la estructura educativa y la agencia del profesor (Giddens, 1991). Así, se aprecian varias formas de actuación según se entienden los roles del profesor.

\section{Resultado 1}

Con relación al primer objetivo, se indican seis tipos de roles para el grupo de participantes; sobresalen dos roles: Orientador y Formador. En la Tabla 1 el número se refiere al conteo de la cantidad de enunciados que aparecen en el discurso de los profesores, que se agrupan según las categorías definidas. Se han agrupado los roles obtenidos a partir de dos características: los vinculados al contexto social amplio y los vinculados al contexto instruccional o de aula. 


\begin{tabular}{|c|c|c|c|c|c|c|}
\hline Área/Roles & Formador & Acompañante & $\begin{array}{l}\text { Modelo/ } \\
\text { Ejemplo }\end{array}$ & Orientador & Instructor & Diseñado \\
\hline Matemáticas & 3 & 0 & 3 & 7 & 4 & 4 \\
\hline Sociales & 5 & 3 & 0 & 6 & 0 & 1 \\
\hline
\end{tabular}

Tabla 1. Roles por disciplina de acuerdo a un modo de entender la función docente

\section{Vinculados al contexto social amplio}

En este grupo el rol se vincula a factores asociados a los estudiantes y sus condiciones, los cuales aparecen como un "mecanismo" a través del cual los profesores actúan asistiéndolos más allá de la enseñanza académica, hasta la formación y el desarrollo personal. Los docentes se relacionan con un sentido del bienestar general, en el que está implicado "el otro". Los contenidos discursivos expresan un modo de entender la práctica diaria del mundo de la vida, una expresión fenomenológica de la política (Díaz, 2003) y una política del estilo de vida (Giddens, 1991). A continuación, se explica cada rol teniendo en cuenta los enunciados identificados en el discurso de los profesores.

- Formador. Vinculado a lograr el desarrollo personal de los estudiantes en las dimensiones afectiva, social y de proyecto de vida; este rol es independiente al conocimiento disciplinar o temático de los estándares, lineamientos y competencias.

- Acompañante. Vinculado a formas de co-responsabilidad; este rol implica participar en los sentimientos del "otro", supone dar consejo y apoyar emocionalmente de acuerdo con las condiciones en que viven los estudiantes.

- Modelo/Ejemplo. El profesor genera credibilidad, confianza, da ejemplo. Su actuación es un prototipo a seguir.

- Orientador. Guiar al estudiante para que adquiera "herramientas" para la vida.

\section{Vinculados al contexto instruccional}

Estos roles se vinculan a los procesos instruccionales, o del aula, empleados para impartir la formación; se relacionan con aspectos didácticos de la enseñanza.
- Instructor. El profesor considera que su función es instruir, entregar el contenido, "dar la materia".

- Diseñador. El profesor "diseña y desarrolla pedagogías" para enseñar en su clase, tomando en cuenta el contexto del estudiante y procurando su transformación.

\section{Resultado 2}

Como se muestra en la Tabla 2, en algunos casos es posible encontrar varios roles. Los contenidos enunciados por los docentes no son solo de tipo pedagógico, instruccional o didáctico, sino que revelan varias atribuciones, por ejemplo, el profesor de matemáticas 1 es diseñador y orientador.

\begin{tabular}{c|c|c|} 
Profesor & Matemáticas & Ciencias sociales \\
1 & Diseñador-Orientador & Orientador-Formador \\
\hline 2 & Diseñador-Formador & Orientador-Formador \\
\hline 3 & $\begin{array}{c}\text { Instructor-Orientador- } \\
\text { Formador }\end{array}$ & Orientador \\
\hline 4 & Diseñador-Orientador & Orientador \\
\hline 5 & Orientador & Formador \\
\hline 6 & Instructor-Orientador & Formador \\
\hline 7 & Instructor-Orientador- & Diseñador \\
\hline 8 & Modelo/Ejemplo & \\
\hline 9 & Modelo/Ejemplo & Orientador-Acompañar \\
\hline 10 & Formador-Modelo/Ejemplo & Formador-Acompañar \\
\hline
\end{tabular}

Tabla 2. Número de roles atribuido por maestro 
A partir de lo expuesto en la Tabla 2, es posible concluir que los profesores tienen un conjunto múltiple de representaciones sobre el rol profesional: el rol atribuido se asocia al sentido que el profesor asigna a las relaciones con otros y a las estrategias de enseñanza (Levin y He, 2008), es decir, a las condiciones de la situación educativa que dan forma a la naturaleza de las acciones del profesor, así como al hecho de ser docentes. En términos de Novoa (2000), el rol aparece como resultado de definiciones sociales, políticas, normas, reglas, creencias, convicciones y verdades acerca de lo que significa ser un buen maestro.

Por otro lado, es evidente que los profesores tienen en común, en ambos enfoques disciplinares, el hecho de que su interés fundamental es formar y orientar, pues son las categorías que prevalecen; con lo cual, buscan guiar al estudiante para que adquiera "herramientas" que le permitan enfrentar la vida y lograr el desarrollo personal en las dimensiones afectiva, social y de proyecto de vida. Esto indica que los profesores creen que la actividad profesional se debe dirigir hacia el desarrollo de fortalezas para enfrentar un entorno social demandante y complejo, cuestión que se ha demostrado en estudios como el de Ottekin (2006).

\section{Conclusiones}

Se han ubicado los roles referenciados en dos grupos: el primero que se refiere al contexto social, y el segundo relacionado con el contexto instruccional. En ambos casos, el estudiante y sus condiciones sociales son el factor central del contexto del cual emergen los roles, sobresaliendo además dos tipos de roles: el de orientadores y facilitadores.

Estos roles suponen un fuerte interés en la formación personal, en ayudar a construir mejores condiciones de vida para los estudiantes y a su vez dotarlos de habilidades que les permitan enfrentarla; no se ve en los docentes un agenciamiento afín a exigencias como las políticas educativas u otras condiciones del sistema educativo, sino a la percepción sobre lo que se necesita para un mejor modo de vida, en términos de Giddens (1991), del estilo de vida que se elige desde la reflexividad personal sobre un contexto diverso.

La reflexividad es un concepto que supone que el rol emerge de la misma capacidad del profesor para monitorear su conducta, y su teoría está en relación con otros actores, lo que algunos teóricos consideran como lo político, la cualidad que se construye o emerge en toda interrelación humana (Díaz, 2003).

En tanto componente de la identidad, el rol es una atribución personal relacionada con la función, delimitada por condiciones contextuales, y sujeta a la interacción docente y a la realidad educativa. De acuerdo con Giddens (1991), el yo no es una entidad pasiva determinada por influjos externos; al forjar sus propias identidades los docentes intervienen con sus cursos de acción de manera directa, lo que hace el profesor está en función de una responsabilidad a cumplir (Badía y Álvarez, 2010). Esta es una característica vinculada a las atribuciones subjetivas sobre la función, que, así, va más allá de las funciones y competencias asignadas para el ejercicio de su actividad en una institución escolar o de las imposiciones políticas de lo que se espera del profesional docente; como se ha indicado, el rol es en cierta medida "asignado", pero a su vez implica un posicionamiento del profesor.

Por lo tanto, indica que el profesor puede ser capaz de acciones políticas desde su interés por la promoción de saberes y de sujetos autónomos (Martínez, 2006); el profesor cuenta con la voluntad para intervenir en las decisiones sobre lo educativo, en los ámbitos de su influencia inmediata; allí está el impacto, en la vida del estudiante, como dice el profesor de sociales PSC7 ${ }^{3}$ : "[...] es transformador porque hay estudiantes que vienen de otros contextos vulnerables y han venido siendo agredidos, subvalorados, y cuando uno les va dando importancia, los transforma, dejándoles ver que son importantes, valiosos, que son inteligentes" (PSC7).

En el profesor los roles se revelan como intereses que van más allá del deber impuesto por el sistema educativo, aparecen ligados a aspectos identitarios, a creencias relacionadas con el impacto de la función, es decir, al beneficio de ejercer esa función:

\footnotetext{
"Eso que te digo es el trabajo de un profesor, su trabajo es tratar de que las personas tengan herramientas que les vayan a servir para su vida futura. Entonces hay personas a las que se les ha dificultado muchas cosas, a todos, a cada uno de nosotros se nos dificulta algo. Entonces, el trabajo del profesor es tratar de dar esas herramientas, a pesar de que se me dificulta mucho utilizarlas, decirme, mire, esta se utiliza así, en tal..., entonces uno, de profesor, debe entender que hay heterogeneidad de personas con dificultad, uno debe tratar de llegar a esos grupos de personas (PM7P4).
}

3 De esta forma se le llamó al profesor entrevistado número 7, de Ciencias Sociales. 
Se encontró que los profesores interactúan con su realidad desde un proceso de ida y vuelta: factores estructurales como la vida de los estudiantes y las condiciones sociales de su entorno o la propia familia, son la fuente de sus decisiones. Los estudiantes son el pivote que los conecta, no son las obligaciones legales; como dice Giddens (1991), la modernidad se ha de entender en un plano institucional, pero los cambios procurados por las instituciones modernas se entretejen directamente con la vida individual y, por tanto, con el yo.

Al examinar el rol desde la perspectiva identitaria, todo indica que para transformar la escuela, y el sistema escolar, es indispensable cambiar de estrategia, es decir, modificar el modo tradicional de pensar y de hacer las reformas e innovaciones que involucran a los docentes. Por más que se actualicen las propuestas curriculares y se implementen programas para la equidad, calidad y universalidad, si no se reconoce en los docentes el factor central del cambio, este no tendrá lugar. Es necesario lograr reformas significativas, efectivas y ante todo sustentables en las prácticas profesionales y culturales del trabajo de los docentes (Vaillant, 2010). En especial, si los roles expresados por los profesores aparecen como la síntesis del proceso de negociación personal, entre lo impuesto y la capacidad de acción política del maestro.

La función docente no es una actividad sujeta únicamente a criterios didácticos e instruccionales enmarcados en el orden jurídico, es también multidimensional; desde el propio docente tiene muchas lecturas, tal vez no explícitas. Para pensar la calidad educativa, es necesario reconocer dimensiones que están más allá de las estrategias de enseñanza, tratando de recoger primero las posturas identitarias de los profesores y la forma en que pueden entrar en contravía diversos aspectos de reforma cuando se insertan las políticas.

Una limitación de la aproximación realizada en esta investigación, es que solo se explora el rol docente desde sus enfoques sobre la función y no desde lo que hace, con lo cual, al no contrastarse con observaciones, no se tiene certeza de la forma en que se evidencian estas disposiciones. Sin embargo, se pueden tomar como indicador para impulsar políticas que permitan a los profesores retomar su capacidad de generar procesos y no ser solamente llevados por estos.

Finalmente, el rol es, de alguna manera, una atribución sobre el oficio; su significado, las formas de vivirlo y las experiencias vinculadas a él son dependientes de las decisiones del sujeto (Eccles, 2009). Una disposición a la acción que se puede usar favorablemente en procesos de formación, comprometiendo al profesor en comunidades de diálogo que inviten a la reflexión sobre la forma de incidir en el bien colectivo, a partir de la reflexión sobre la finalidad de la actividad profesional del profesor en la educación secundaria. 


\section{Referencias}

Akkerman, S. F., y Meijer, P. C. (2010). A Dialogical approach to conceptualizing teacher identity. Teaching and Teacher Education, pp. 1-12.

Akerlind, G. S. (2005). Variation and commonality in phenomenographic research methods. Higher Education Research \& Development, 24 (4), pp. 321-334.

Arancibia, M. (2011). Concepciones del profesor sobre aprender y enseñar historia y su relación con tipos de usos educativos de las TIC. Tesis doctoral (Sin publicar). Barcelona.

Arriaga, J. M. (2013). Impacto de las políticas educativas en la identidad docente. Revista Digital. Mundialización Educativa, 5. Obtenido desde http://ece.edu.mx/ecedigital/files/ ArticuloJuany.pdf

Badia, A., y Álvarez, I. (2010). La identidad del profesor en contextos de educación presencial y virtual. En Monereo, C., y Pozo, J. (2011) (Eds.). La identidad en Psicología de la Educación. Necesidad, utilidad y límites. (pp., 213-233). Madrid: Narcea.

Badia, A., y Gómez, F. (2014). Educational context conditions affecting the teaching design of University teacher. Educación XXI, 17 (2), pp. 169-192.

Ben-Peretz, M., Mendelson, N., y Kron, W. F. (2003). How teachers in different educational context view their roles. Teaching and Teacher Education, 19, pp. 277-290.

Beauchamps, C., y Thomas, L. (2009). Understanding teacher identity: an overview of issues in the literature and implications for teacher education, 39(2), pp. 175-189.

Beijaard, D., Meijer, P., y Verloop, N. (2004). Reconsidering research on teacher's'professional identity. Teaching and teacher education, 20, p. 107-128.

EdUCACIÓN Y CIUdAd No 29 JULIO - DiCIEMBRE DE 2015 ISSN 0123-0425 - PP 188 - 198
Bolívar, A. (2006). La identidad profesional del profesorado de secundaria: crisis y reconstrucción. Málaga: Aljibe.

Carvajal, E., y Gómez, M. (2002). Concepciones y representaciones de los maestros de secundaria y bachillerato sobre la naturaleza, el aprendizaje y la enseñanza de las ciencias. Revista Mexicana de Investigación Educativa. 7(16), pp. 577-602.

Cohen, J. (2010). Getting recognized: Teachers negotiating professional identities as learners through all. Teaching and Teacher Education, 26, pp. 473481

Day, C., Kington, A., Stobart, G., y Sammons, P. (2006). The personal and professional selves of teachers: stable and unstable identities. British Educational Research Journal, 32(4), pp. 601-616.

Day, C. (2006). Pasión por enseñar. La identidad personal y profesional del docente y sus valores. Madrid: Narcea.

Díaz, A. (2003). Una discreta diferenciación entre la política y lo político y su incidencia sobre la educación en cuanto socialización política. Reflexión Política, 5(9), pp. 48-58.

Eccles, J. (2009). Who am I and what am a going to do with my life? Personal and colletive identities as motivators of action. Educational Psychologist, 44(2), pp. 78-94.

Engels. A. (2008). Construcción del conocimiento en entornos virtuales de enseñanza y aprendizaje. Tesis doctoral (sin publicar). Programa de doctorado Cultura, educación y sistemas semióticos, Facultad de Psicología. Universidad de Barcelona.

Enyedy, N., Goldberg, J., y Muir W. (2005). Complex dilemmas of identity and practice. Wiley Interscience, pp. 68-93.

Flick, U. (2007). Introducción a la investigación cualitativa. Madrid: Narcea. 
Galaz, A. (2011). El profesor y su identidad profesional ¿Facilitadores u obstáculos del cambio educativo? Estudios pedagógicos, 2(89), p. 107.

Gee, J. P. (2000). Identity as an Analytic Lens for Research in Education. Review of Research in Education, 25, pp. 99-125.

Gil, C. F., y Rico, R. L. (2003). Concepciones y creencias del profesorado de secundaria sobre enseñanza y aprendizaje de las matemáticas. Enseñanza de las Ciencias, 1 (21), pp. 27-47.

Giddens, A. (1991). Modernity and self. Identity, self and society in the late modern age. California: Stanford University press.

Hermans, J. M. (2001). The Dialogical Self: Toward a Theory of Personal and Cultural Positioning. Culture \& Psychology, 7(3), pp. 243-281.

Hamman, D., Gosselin, K., Romano, J., y Bunuan, R. (2010). Using possible-selves theory to understand the identity development of new teachers. Teaching and Teacher Education. DOI:10.1016/j. tate.2010.03.005

Kelly, O., Dorf, H., Pratt, N., y Hohmann, U. (2013). Comparing teacher roles in Denmark and England. Compare: A journal of comparative and international education. DOI:10.1080/0305792 5.2013 .800786 .

Lasky, S. (2005). A sociocultural approach to understanding teacher identity, agency and professional vulnerability in a context of secondary school reform. Teaching and Teacher Education, 21, p. 899 .

Levin, B., y He, Y. (2008). Investigating the content and sources of teacher candidates personal practical theories (ppts). Journal of teacher education, 59 (1), pp. 59-68.

Lechner, N. (1986). Especificando la política. La conflictiva y nunca acabada construcción del orden deseado. Madrid: Siglo XXI.

Marton, F. (1994). Phenomenography. En Husen, T., y Postlethwaite, T. N. (Ed.). The international encyclopedia of education. Oxford: Elsevier Science, pp. 4424-4429.

Marton, F., y Pang, M. F. (1999). Two faces of variation. Paper presented at the $8^{\text {th }}$ European conference for learning and instruction. Goteborg, Sweden.
Martínez, M. C. (2006). La figura del maestro como sujeto político: El lugar de los colectivos y redes pedagógicas en su agenciamiento. Educere, 10(33), pp. 243-250. Obtenido desde: http://www.redalyc.org/pdf/356/35603305.pdf

Monereo, C. (2010). La formación del profesorado: Una pauta para el análisis e intervención a través de incidentes críticos. Revista Iberoamericana de educación, 52, pp. 149-162.

Monereo, C., y Badía, A. (2011). Los heterónimos del docente. Identidad, selfs y enseñanza. En Monereo, C., y Pozo, J. (Eds.). La identidad en Psicología de la Educación. Necesidad, utilidad y límites. (pp. 57-76). Madrid: Narcea.

Monereo, C., y Monte, M. (2011). Docentes en tránsito. Incidentes críticos en secundaria. Barcelona: Grao.

Nargund-Joshi, V., Rogers, M., y Akerson, V. (2011). Exploring Indian secondary teachers' orientations and practice for teaching science in an era of reform. Journal of Research in Science Teaching, 48 (6), pp. 624-647.

Novoa, A. (2000). Ways of saying, ways of seeing-public images of teachers (19th-20th centuries). Pedagogica Historica, 36(1), pp. 21-52.

O'Connor, K. (2008). "You Choose to care": Teachers emotions and professional identity. Teaching and Teacher Education, 24, pp. 117-126.

Ottekin, A. (2006). Education faculty students' tendencies and beliefs about the teacher's role in education: A case study in a Turkish University. Teaching and Teacher Education, 22, pp. 1068-1083.

Salazar, R. E., Valenzuela, B., Cortés, G. E., y Guillén, M. (2013). La identidad docente, la política y gobernabilidad en educación superior. European Scientific Journal, 9 (2), pp. 1857-7431.

Settlage, J., Southerlan, S., Smith, L., y Ceglie, R. (2009). Constructing a Doubt-free teaching self: Self-Efficacy, teacher identity, and science instruction within diverse settings. Journal of Research in Science Teaching, 46(1), pp. 102-125.

Stillwagon, J. (2008). Performing for the Students: Teaching Identity and the Pedagogical Relationship. Journal of Philosophy of Education, 42(1), pp. 67-83. 
Trickett, E. J., Rukhotskiy, E., Jeong, A., Genkova, A., Oberoi, A., Weinstein, T., y Delgado, Y. (2012). The kids are terrific: It's the job that's tough: The ELL teacher role in an urban context. Teaching and Teacher Education, 28, pp. 283-292.

Van Veen, K. y Sleegers, P. (2006). How does it feel? Teachers' emotions in a context of change. Journal of Curriculum Studies, 38 (1), pp. 85-111.

Van Veen, K., Sleegers, P., y Van de Ven P-H. (2005b). One teacher's identity, emotions, and commitment to change: A case study into the cognitive-affective processes of a secondary school teacher in the context of reform. Teaching and Teacher Education, 21, pp. 917-934.

Vaillant, D. (2010). La identidad docente. La importancia del profesorado. Revista Novedades Educativas, 234

Waeytens, K., Lens, W., y Vandenberghe, R. (2002). "Learning to learn": Teachers'conceptions of their supporting role. Learning and Instruction, 12, pp. 305-322.

Weise, C. (2011). La atención a contextos de alta diversidad sociocultural en la Universidad. Un análisis de la identidad y la práctica docente a través de incidentes críticos. Tesis doctoral (sin publicar). Obtenido desde http://www.tdx.cat/bitstream/handle /10803/129079/ccc1de1.pdf;jsessionid=B20F5C3DBB796AE444A9B9C07DBE29E8.tdx2? sequence=1

Wenger, E. (2001). Comunidades de práctica: Aprendizaje, significado e identidad. Barcelona: Paidós.

Wertsch, J. (1991). Voices of mind: A sociocultural approach to mediated action. Cambridge: Harvard University Press.

Zembylas, M. (2005). Discursive practices genealogies, and emotional rules: a postestructuralist view on emotion and identity in teaching. Teaching and Teacher Education, 2, pp. 919-935.

Zucker, L. (1987). Institutional Theories of organization. Annual Review of Sociology, 13, pp. 443-464. 